\title{
The oxygen sensor PHD3 limits glycolysis under hypoxia via direct binding to pyruvate kinase
}

\author{
Cell Research (2011) 21:983-986. doi:10.1038/cr.2011.66; published online 12 April 2011
}

\section{Dear Editor,}

Oxygen levels are critical for cellular physiology. Under low oxygen (hypoxia), cells increase glycolysis while limiting mitochondrial oxidative metabolism. Such adaptation is largely mediated by the transcription factors HIF, which accumulate under hypoxia $[1,2]$. Prolyl hydroxylase domain proteins (PHD) function as cellular oxygen sensors and target HIF for subsequent degradation under normoxia [2]. The PHD/HIF pathway is conserved in Drosophila, where a single prolyl hydroxylase, Fatiga, has been characterized. We have previously reported that Fatiga regulates cellular growth rates in a HIF-independent manner, yet mechanisms remained elusive [3].

To characterize novel mechanisms controlling growth and metabolism in response to oxygen sensing, we immunoprecipitated HA-tagged Fatiga from Drosophila S2 cells and detected interacting proteins by mass spectrometry (Supplementary information, Table S1 and Data S1). We identified Sima, the fly HIF- $\alpha$ ortholog, as well as several components of the T-complex chaperon proteins (TriC), whose mammalian homologs interact with PHD3 [4]. In addition, we identified PyK, encoding the glycolytic enzyme pyruvate kinase (PK; EC 2.7.1.40), which produces pyruvate from phosphoenolpyruvate (PEP) (Figure 1A). Importantly, a Fatiga hydroxylase-deficient mutant still interacted with PyK, but lost its interaction to Sima (Figure 1B). To test whether the PyK-Fatiga interaction would be conserved in mammalian proteins, we used the M2 isoform of human PK (PK-M2) in pulldown assays. Indeed, PK-M2 interacted with PHD3, but not PHD1 or PHD2 (Figure 1C). As proof of this interaction in vivo, endogenous PK-M2 and PHD3 did co-immunoprecipitate in mouse embryonic fibroblasts (MEFs; Figure 1D).

In mammals, four isoenzymes of PK are found: type L in liver, R in erythrocytes, M1 in adult and M2 in embryonic cells. During tumorigenesis, tissue-specific isoforms of PK are replaced by PK-M2, and this isoform plays an important role in the metabolic shift of cancer cells [5, 6]. PK-M2 is a tetramer in the presence of FBP (fructose 1,6-bisphosphate), showing a high affinity to its substrate PEP $\left(\mathrm{K}_{\mathrm{m}} 0.17 \mathrm{mM}\right)$. In the absence of FBP, PK-M2 exists as a dimer or monomer, and its affinity to PEP is much lower $\left(\mathrm{K}_{\mathrm{m}} 2.1 \mathrm{mM}\right)$ [7]. In tumor cells, PK-M2 exists mainly as dimer/monomer and is believed to be inactive under the physiological PEP concentration [6]. To distinguish different PK-M2 forms, we measured PK activity using different concentrations of PEP. Indeed, activity of GST-PK-M1 was less sensitive to reduction in PEP concentration (from $5 \mathrm{mM}$ to $0.2 \mathrm{mM}$ ) than GST-PK-M2 (Supplementary information, Figure S1A). Similarly, overexpressed PK-M2 was more sensitive to decreased PEP concentration than PK-M1 (Supplementary information, Figure S1B and S1C). Furthermore, whereas GSTPK-M1 was insensitive to FBP stimulation, activity of GST-PK-M2 could be stimulated by FBP at low PEP concentration $(0.2 \mathrm{mM})$ but not at high PEP concentration (5 mM; data not shown). These data show that PK activity in response to different PEP concentrations can be used to distinguish between the M1-like behavior of M2 (constant tetramer form) and the M2 behavior (equilibrium between tetramer and dimer/monomer form).

Next, we knocked down PHD3 levels in HeLa cells using a specific shRNA, which led to a strong down-regulation of endogenous PHD3 mRNA and protein levels (Supplementary information, Figures S2 and Figure 1E). Since PHD3 protein levels accumulate under hypoxia, we grew cells under normoxic as well as hypoxic conditions to test for an effect on PK activity. The shRNA ${ }^{\text {PHD3 }}$ cells showed no change in PK activity when assayed under high PEP conditions (5 mM), which detects all forms of PK, but had higher PK activity when using low PEP concentration $(0.2 \mathrm{mM})$, which specifically assays the tetramer form of PK-M2. This effect was seen in cells exposed to hypoxia. Furthermore, PK activity of shRNA ${ }^{\text {PHD3 }}$ cells was insensitive to FBP, which is known to promote tetramer formation of PK-M2 (Figure 1F and $1 \mathrm{G}$ ). To directly assess various forms of PK-M2, we used Native Blue gels. When hypoxia-treated shRNA${ }^{\text {Control }}$ lysates were analyzed, monomeric and dimeric PKM2 was predominant, and only 5\% of PK-M2 existed 


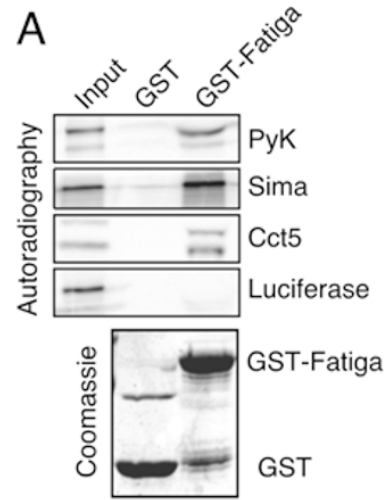

$\mathrm{F}$ Relative PK activity
B

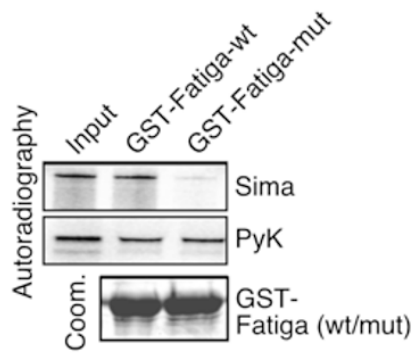

C

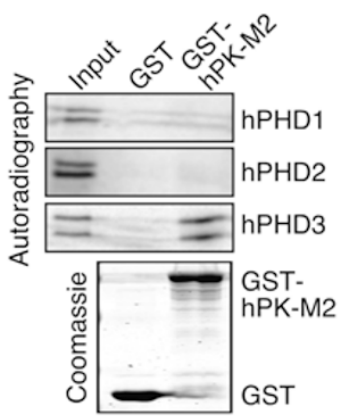

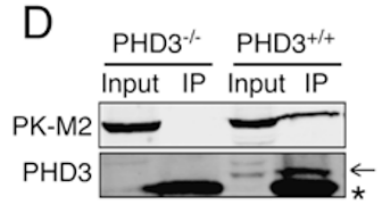

$E$

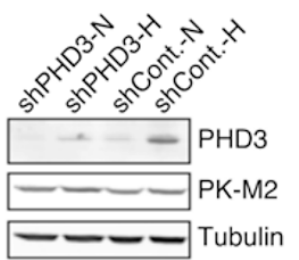

$\mathrm{G}$

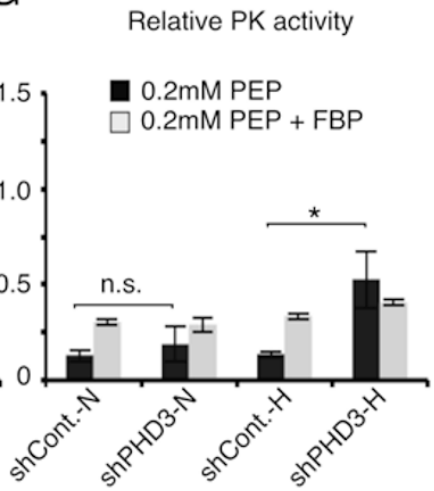

$\mathrm{H}$

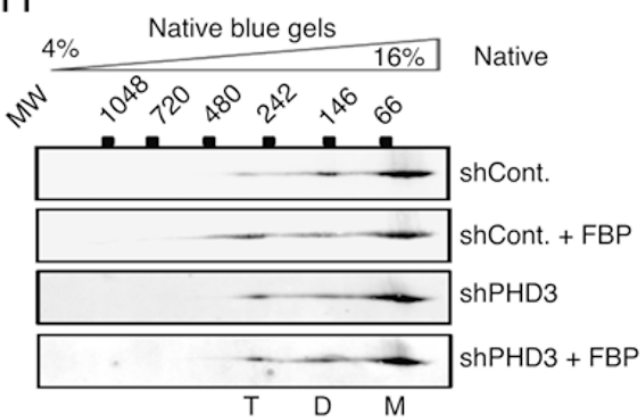

$\mathrm{K}$
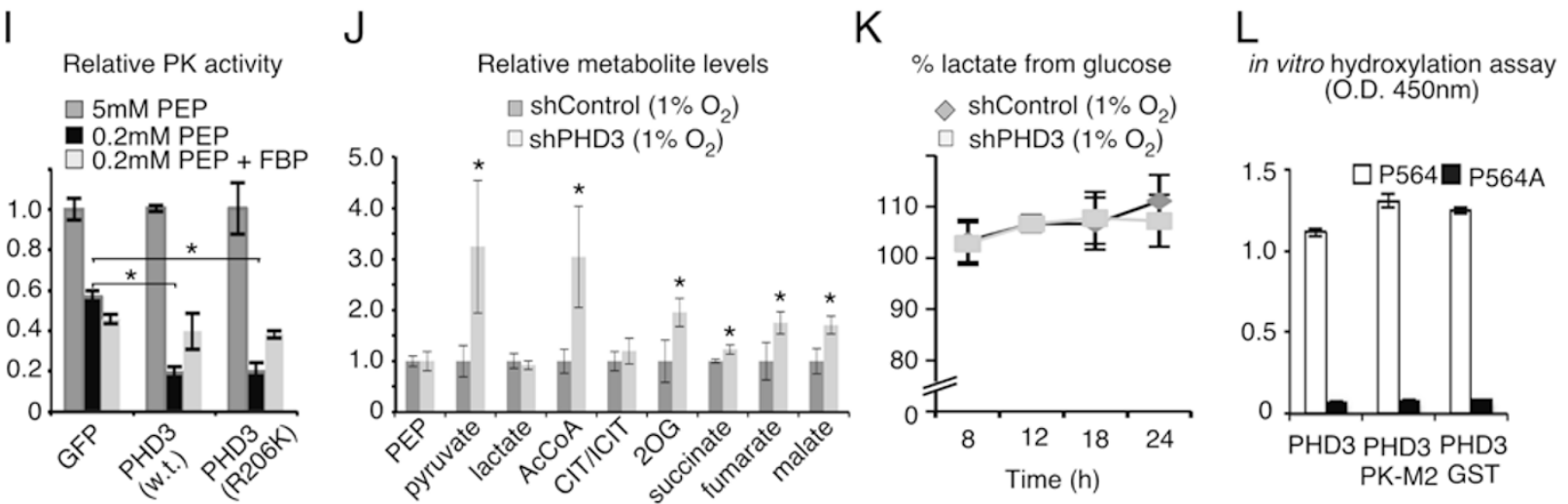

Figure 1 PHD3 interacts with PK-M2 and inhibits PK activity. (A) In vitro translated PyK, Sima, Cct5 or control Luciferase were pulled-down by GST or GST-Fatiga and detected by autoradiography. (B) PyK or Sima was pulled-down by GSTFatiga-wt (wild type) or GST-Fatiga-mut $\left(\mathrm{H}^{169} \mathrm{~A}, \mathrm{D}^{171} \mathrm{~A}\right.$ and $\mathrm{H}^{230} \mathrm{~A}$ triple mutant). (C) Human PHD proteins were pulled-down by GST or GST-hPK-M2. In A-C, GST and GST-fusion proteins are shown on coomassie-stained SDS-PAGE gels. (D) MEFs from PHD3 $^{-/}$or wild-type mice were treated with $1 \% \mathrm{O}_{2}$ for $16 \mathrm{~h}$. PHD3 was immunoprecipitated and western blotting was carried out to detect PHD3 and PK-M2. The arrow indicates PHD3 and the star indicates IgG light chain. (E) HeLa cells stably transfected with shRNA ${ }^{\text {control }}$ or shRNA ${ }^{\mathrm{PHD} 3}$ were exposed to normoxia $(\mathrm{N})$ or hypoxia $\left(\mathrm{H} ; 1 \% \mathrm{O}_{2}\right.$ for $\left.16 \mathrm{~h}\right)$. Protein levels of PHD3 and PK-M2 were measured by western blotting. (F, G) Pyruvate kinase activities from whole cell lysates were measured using the indicated PEP concentration. For $\mathbf{F}$ and $\mathbf{G}$, activity of normoxic shRNA ${ }^{\text {control }}$ samples using 5 mM PEP was set to 1. (H) Whole cell lysates from hypoxia-treated cells were separated using Native Blue gels. Monomeric (M; 58 $\mathrm{kDa}$ ), dimeric ( $\mathrm{D} ; 116 \mathrm{kDa}$ ) and tetrameric forms (T; $232 \mathrm{kDa}$ ) of PK-M2 were detected by western blotting. (I) The shRNA ${ }^{\text {PHD3 }}$ cells were transfected with V5 tagged GFP or PHD3 rescue constructs (wild type or R206K mutant) and treated with hypoxia. PK activity was measured as in $\mathbf{F}$ and $\mathbf{G}$. ( $\mathbf{J}$ ) Metabolites of hypoxia-treated cells were extracted and measured by LC-MS/MS. AcCoA: Acetyl-CoA; CIT/ICIT: Citrate/isocitrate; 2OG: 2-oxoglutarate. (K) A mixture of ${ }^{13} \mathrm{C} /{ }^{12} \mathrm{C}$-glucose was added to hypoxia-treated shRNA ${ }^{\text {control }}$ or shRNA ${ }^{\mathrm{PHD} 3}$ cells $\left(1 \% \mathrm{O}_{2}\right.$ for $\left.18 \mathrm{~h}\right)$. At the indicated time point, secreted lactate was analyzed by LC-MS/MS for the ${ }^{13} \mathrm{C} /{ }^{12} \mathrm{C}$-ratio. Shown is the percentage of lactate that is derived from glucose. (L) In vitro assay using PHD3 purified from Sf9 cells. As a substrate, a HIF-1 $\alpha$ peptide was used (aa 556-574 wild type or P564A mutant). Purified recombinant PK-M2 or GST was used in equimolar concentrations compared to PHD3. All error bars denote standard deviation $(n \geq 3)$. 
as tetramer. Incubation with FBP stimulated tetramer formation (to $27 \%$ ). Importantly and agreeing with the activity measurements described above, down-regulation of PHD3 increased the tetramer ratio to $26 \%$ even in the absence of FBP, which could not be further stimulated by FBP (Figure 1H and Supplementary information, Figure S3). To prove that the effect seen on PK activity was caused by the down-regulation of PHD3, we re-expressed an shRNA-resistant form of PHD3. Indeed, the increase in PK activity of shRNA ${ }^{\mathrm{PHD} 3}$ cells was completely rescued by exogenous PHD3, and PK activity could again be stimulated by FBP (Figure 1I and Supplementary information, Figure S4). Moreover, a hydroxylase-dead PHD3 (R206K) showed an identical rescue (Figure 1I and Supplementary information, Figure S4), supporting that PHD3 controls the PK-M2 equilibrium between the tetramer, dimer and monomer forms in a hydroxylaseindependent manner.

Since down-regulation of PHD3 led to increased PK activity at physiological PEP concentrations, we measured cellular metabolite levels from shRNA ${ }^{\text {Control }}$ and shRNA ${ }^{\text {PHD3 }}$ cells at hypoxic conditions. PHD3 downregulation resulted in a 3-fold increase in pyruvate levels, which is consistent with the higher PK activity in these cells (Figure 1J). Remarkably, the increase in pyruvate did not lead to enhanced synthesis of lactate (both intracellular and secreted), but led to more acetyl-CoA and the TCA-cycle intermediates 2-oxoglutarate, succinate, fumarate and malate (Figure $1 \mathrm{~J}$ and data not shown). In addition to glucose, cells take up glutamine and convert it to TCA cycle intermediates, pyruvate and lactate through the process of glutaminolysis. To test whether pyurvate could be derived from sources other than glucose, we grew $\mid$ cells in a medium containing ${ }^{13} \mathrm{C}$-glucose, and used mass spectrometry to determine the carbon source of lactate. For both shRNA ${ }^{\text {Control }}$ and shRNA ${ }^{\text {PHD3 }}$ cells, lactate was entirely derived from glucose (Figure $1 \mathrm{~K}$ ), thus strongly supporting our conclusion that the increase in pyruvate is due to enhanced PK activity upon knockdown of PHD3. To further test whether the effect seen on PK activity would alter the cell's capacity to respond properly to hypoxia, we performed two additional assays. First, we measured reactive oxygen species (ROS), which are mostly derived from mitochondrial oxidative phosphorylation. Upon hypoxia, down-regulation of PHD3 led to a significant increase in ROS (Supplementary information, Figure S5A). Finally, we performed proliferation assays. Upon down-regulation of PHD3, cells divided at a lower rate when grown at $3 \% \mathrm{O}_{2}$, but not under normoxia (Supplementary information, Figure S5B), demonstrating that PHD3 is critical for cells to adapt properly to hypoxia.

Taken together, we demonstrate that PHD3 interacts with PK-M2, leading to inhibition of PK activity in vivo. Since PHD3 protein is less abundant under normoxia but increases under hypoxia, the effects seen are largely hypoxia-specific. Regulation of PK-M2 activity is achieved by shift of equilibrium between tetramer and dimer/monomer forms. Herein we propose that the binding of PHD3 to PK-M2 either leads to dissociation of the PK-M2 tetramers, or impedes the formation of tetramers from monomers/dimers. The effect on PK activity is independent of PHD3's hydroxylation activity, strongly suggesting that PK-M2 is not a novel hydroxylation target. Even though PHD2, rather that PHD3, is the major hydroxylase that targets HIF in vivo [8], we tested whether the knockdown of PHD3 would affect HIF activity. Importantly, mRNA levels of several known HIF target genes, including PK-M2, were not affected upon PHD3 knockdown (Supplementary information, Figure S2), supporting the model that PK-M2 is regulated by PHD3 via protein interaction. To test whether PHD3 might be altered in response to PK-M2 binding, we performed an in vitro assay to detect PHD3's hydroxylation activity towards a HIF peptide [9]. Importantly, PK-M2 had no effect (Figure 1L), suggesting that PHD3 is not regulated by PK-M2, at least in vitro. Since tumors are often hypoxic and predominantly express the PK-M2 isoform, the PHD3/PK-M2 interaction might be particularly important in tumors. Accordingly, recent data showed that PHD3 protein levels were up-regulated in pancreatic tumors and increased levels correlated with aggressive tumor behavior [10]. Since PHD3 engages direct proteinprotein interaction with $\mathrm{PK}$, it can therefore give more immediate response, and presumably in a reversible way to adjust metabolism according to oxygen availability. In the future, pharmacological interference with the PHD3/ PK-M2 interaction could therefore be used as a novel strategy to reduce tumor growth.

\section{Acknowledgments}

We acknowledge M Cockman and P Ratcliffe (University of Oxford, UK) for the generous gift of the PHD3 antibody and cell lines, and Roland Scholz (ETH Zürich, Switzerland) for technical help. This work was supported by the BEST cluster for Bioengineering (ETH Zurich), the Roche Research Foundation (Mkkl/stm 86-2008) and the Swiss National Science Foundation (PP00A-106737 and PP00P3 123359/1-2; all to CF).

Nan Chen ${ }^{1,2}$, Oliver Rinner ${ }^{1}$, Dominika Czernik ${ }^{1}$, Katarzyna J Nytko ${ }^{3}$, Dan Zheng ${ }^{1}$, Daniel P Stiehl ${ }^{3}$, Nicola Zamboni ${ }^{1}$, Matthias Gstaiger ${ }^{1}$, Christian Frei ${ }^{1}$

${ }^{I}$ ETH Zurich, Department of Biology and Competence Center for Systems Physiology and Metabolic Diseases (CC-SPMD), 
8093 Zurich, Switzerland; ${ }^{2}$ Laboratory of Physical Biology, Shanghai Institute of Applied Physics, Chinese Academy of Sciences, Shanghai 201800, China; ${ }^{3}$ University of Zurich, Institute of Physiology and Zurich Center for Integrative Human Physiology (ZIHP), 8057 Zurich, Switzerland

Correspondence: Christian Frei

Tel: +41 4463333 94; Fax: +41 446331069

E-mail: christian.frei@cell.biol.ethz.ch

\section{References}

1 Gatenby RA, Gillies RJ. Why do cancers have high aerobic glycolysis? Nat Rev Cancer 2004; 4:891-899.

2 Kaelin WG Jr, Ratcliffe PJ. Oxygen sensing by metazoans: the central role of the HIF hydroxylase pathway. Mol Cell 2008; 30:393-402.

3 Frei C, Edgar BA. Drosophila cyclin d/cdk4 requires hif-1 prolyl hydroxylase to drive cell growth. Dev Cell 2004; 6:241251.

4 Masson N, Appelhoff RJ, Tuckerman JR, et al. The HIF prolyl hydroxylase PHD3 is a potential substrate of the TRiC chape- ronin. FEBS Lett 2004; 570:166-170.

5 Christofk HR, Vander Heiden MG, Harris MH, et al. The M2 splice isoform of pyruvate kinase is important for cancer metabolism and tumour growth. Nature 2008; 452:230-233.

6 Mazurek S. Pyruvate kinase type M2: A key regulator of the metabolic budget system in tumor cells. Int J Biochem Cell Biol 2010 Feb 13; doi:10.1016/j.biocel.2010.02.005

7 Dombrauckas JD, Santarsiero BD, Mesecar AD. Structural basis for tumor pyruvate kinase M2 allosteric regulation and catalysis. Biochemistry 2005; 44:9417-9429.

8 Berra E, Benizri E, Ginouves A, et al. HIF prolyl-hydroxylase 2 is the key oxygen sensor setting low steady-state levels of HIF-1alpha in normoxia. EMBO J 2003; 22:4082-4090.

9 Nytko KJ, Spielmann P, Camenisch G, Wenger RH, Stiehl DP. Regulated function of the prolyl-4-hydroxylase domain (PHD) oxygen sensor proteins. Antioxid Redox Signal 2007; 9:13291338.

10 Couvelard A, Deschamps L, Rebours V, et al. Overexpression of the oxygen sensors PHD-1, PHD-2, PHD-3, and FIH Is associated with tumor aggressiveness in pancreatic endocrine tumors. Clin Cancer Res 2008; 14:6634-6639.

(Supplementary information is linked to the online version of the paper on the Cell Research website.) 